\title{
MIRROR THEOREM FOR ELLIPTIC QUASIMAP INVARIANTS
}

\author{
BUMSIG KIM AND HYENHO LHO
}

\begin{abstract}
We propose and prove a mirror theorem for the elliptic quasimap invariants of smooth Calabi-Yau complete intersections in projective spaces. The theorem combined with the wall-crossing formula appeared in 3 implies mirror theorems of Zinger and Popa for the elliptic Gromov-Witten invariants of those varieties. This paper and the wall-crossing formula provide a unified framework for the mirror theory of rational and elliptic Gromov-Witten invariants.
\end{abstract}

\section{INTRODUCTION}

Let $W$ be a codimension $r$ affine subvariety in $\mathbb{C}^{n}$ defined by homogeneous degree $l_{1}, \ldots, l_{r}$ polynomials such that the origin is the only singular point of $W$. Assume

$$
\sum_{a=1}^{r} l_{a}=n
$$

and let $\mathbf{G}:=\mathbb{C}^{*}$ act on $\mathbb{C}^{n}$ by the standard diagonal action so that its associated GIT quotient

$$
X:=W / / \mathbf{G}
$$

is a codimension $r$, nonsingular Calabi-Yau complete intersection in $\mathbb{P}^{n-1}$.

With this setup, for each positive rational number $\varepsilon$ there are socalled $\varepsilon$-stable quasimap moduli space

$$
Q_{g, 0}^{\varepsilon}(X, d)
$$

with the canonical virtual fundamental class $\left[Q_{g, 0}^{\varepsilon}(X, d)\right]^{\text {vir }}$ (see [6]). We are mainly interested in the space $Q_{g, 0}^{\varepsilon}(X, d)$ with small enough $\varepsilon$ with respect to degree $d$, which will be denoted by $Q_{g, 0}^{0+}(X, d)$ and also simply by $Q_{g, 0}(X, d)$. When $\varepsilon>2, Q_{g, 0}^{\varepsilon}(X, d)$ coincides with the moduli space $\bar{M}_{g, 0}(X, d)$ of stable maps, which will be denoted also by $Q_{g, 0}^{\infty}(X, d)$. 
When $g=1$, the virtual dimension of $Q_{g, 0}^{\varepsilon}(X, d)$ is always zero. The main goal of this paper is to discover an explicit description of $\operatorname{deg}\left[Q_{1,0}(X, d)\right]^{\text {vir }}$ in terms of the Givental's $I$-function for $X$. Let

$$
\langle\rangle_{1,0}^{\varepsilon}:=\sum_{d=1}^{\infty} q^{d} \operatorname{deg}\left[Q_{1,0}^{\varepsilon}(X, d)\right]^{\text {vir }},
$$

where $q$ is a formal Novikov variable. We express the generating function \langle\rangle$_{1,0}^{\varepsilon}$ in terms of Givental's T-equivariant $I$-function for $X$, where $\mathbf{T}:=\left(\mathbb{C}^{*}\right)^{n}$ is the complex torus group acting on $\mathbb{P}^{n-1}$ (see [7]).

The equivariant $I$-function is the $H_{\mathbf{T}}^{*}\left(\mathbb{P}^{n-1}\right) \otimes \mathbb{Q}(\lambda)$-valued formal function in formal variables $q, z, t_{H}$ :

$$
I_{\mathbf{T}}(t, q):=e^{t_{H} H / z} \sum_{d=0}^{\infty} q^{d} e^{t_{H} d} \frac{\prod_{a=1}^{r} \prod_{k=1}^{l_{a} d}\left(l_{a} H+k z\right)}{\prod_{k=1}^{d} \prod_{j=1}^{n}\left(H-\lambda_{j}+k z\right)},
$$

where $\lambda_{1}, \ldots, \lambda_{n}$ are the T-equivariant parameters; $\mathbb{Q}(\lambda)$ denotes the quotient field of the polynomial ring in $\lambda_{1}, \ldots, \lambda_{n} ; H$ is the $\mathbf{T}$-equivariant hyperplane class; and $t:=t_{H} H$.

Let $\lambda_{0}$ be another formal parameter. Consider the restriction $\left.I_{\mathbf{T}}(0, q)\right|_{p_{i}}$ of $I_{\mathbf{T}}(0, q)$ to the $i$-th $\mathbf{T}$-fixed point

$$
p_{i}:=[\underbrace{0, \ldots, 1}_{i}, \ldots, 0] \in \mathbb{P}^{n-1} .
$$

Define the $q$-series $\mu(q), R_{0}(q) \in \mathbb{Q}[[q]]$ by the asymptotic expansion

$$
\left.I(0, q)\right|_{p_{i}} \equiv e^{\mu(q) \lambda_{i} / z}\left(R_{0}(q)+O(z)\right),
$$

where $\equiv$ means the equality after the specialization

$$
\lambda_{i}=\lambda_{0} \exp (2 \pi i \sqrt{-1} / n), \quad i=1, \ldots, n .
$$

For the existence of the asymptotic expansion, see (2.7). Denote by

\section{$\underline{I}_{\mathbf{T}}$}

the specialization of $I_{\mathbf{T}}$ with (1.3).

For $k=0,1, \ldots, n-1$, define the initial constants $C_{k}(q) \in \mathbb{Q}[[q]]$ of form $1+O(q)$ inductively by the requirements

$$
C_{k}(q) H^{k}=B_{k}+O(1 / z)
$$

in the following Birkhoff factorization procedure:

$$
\begin{aligned}
B_{0}:=\underline{I}_{\mathbf{T}}(0, q), B_{1}:=\left(H+z q \frac{d}{d q}\right) \frac{B_{0}}{C_{0}(q)}, \cdots, \\
B_{k}:=\left(H+z q \frac{d}{d q}\right) \frac{B_{k-1}}{C_{k-1}(q)}, \cdots, B_{n-1}:=\left(H+z q \frac{d}{d q}\right) \frac{B_{n-2}}{C_{n-2}(q)} .
\end{aligned}
$$


There is an interpretation of $C_{k}$ as a $\mathbf{T}$-equivariant quasimap invariant (see Remark 3.3).

Now we are ready to state one of two main results of this paper.

\section{Theorem 1.1.}

$$
\begin{aligned}
&\langle\rangle_{1,0}^{0+}=-\frac{3(n-1-r)^{2}+n-r-3}{48} \log \left(1-q \prod_{a=1}^{r} l_{a}^{l_{a}}\right) \\
&-\frac{1}{2} \sum_{k=0}^{n-2-r}\left(\begin{array}{c}
n-r-k \\
2
\end{array}\right) \log C_{k}(q) .
\end{aligned}
$$

There is a following wall-crossing formula conjectured in [3] and proven in [5]. Define $I_{0}:=C_{0}$ and $I_{1}$ by the $1 / z$-expansion of

$$
\left.I_{\mathbf{T}}\right|_{\lambda=t=0}=I_{0}+I_{1} / z+O\left(1 / z^{2}\right) .
$$

Theorem 1.2. [5]

$$
\begin{aligned}
& \left.\langle\rangle_{1,0}^{\infty}\right|_{q^{d_{\mapsto} \mapsto q^{d}} \exp \left(\int_{d[\text { line }]} \frac{I_{1}}{I_{0}}\right)}-\langle\rangle_{1,0}^{0+} \\
& =\frac{1}{24} \chi_{\mathrm{top}}(X) \log I_{0}+\frac{1}{24} \int_{X} \frac{I_{1}}{I_{0}} c_{\operatorname{dim} X-1}(T X) .
\end{aligned}
$$

Here $\chi_{\mathrm{top}}(X)$ is the topological Euler characteristic of $X$ and $c_{\operatorname{dim} X-1}(T X)$ is the $(\operatorname{dim} X-1)$-th Chern class of the tangent bundle $T X$.

Without any usage of the reduced Gromov-Witten invariants, Theorem 1.1 combined with the wall-crossing formula (1.4) reproves the following mirror theorem of Zinger and Popa for Calabi-Yau complete intersections in projective spaces.

Theorem 1.3. [12, 11]

$$
\begin{array}{r}
\left.\langle\rangle_{1,0}^{\infty}\right|_{q^{d_{\mapsto}} \rightarrow q^{d} \exp \left(\int_{d[\text { line }]} I_{1} / I_{0}\right)}=\frac{1}{24} \chi_{\mathrm{top}}(X) \log I_{0}+\frac{1}{24} \int_{X} \frac{I_{1}}{I_{0}} c_{\operatorname{dim} X-1}(T X) \\
-\frac{3(n-1-r)^{2}+n-r-3}{48} \log \left(1-q \prod_{a=1}^{r} l_{a}^{l_{a}}\right) \\
-\frac{1}{2} \sum_{k=0}^{n-2-r}\left(\begin{array}{c}
n-r-k \\
2
\end{array}\right) \log C_{k}(q) .
\end{array}
$$

The above three theorems are logically independently proven and any pair of them implies the remaining theorem. Theorem 1.1 combined 
with Theorem 1.4 answers the question raised by Marian, Oprea, and Pandharipande in $\S 10.2$ of [10].

The strategy to prove Theorem 1.1 consists of two steps. The first step is quite general and conceptual. We obtain Theorem 2.6. one of two main results of this paper. The theorem is a quasimap version of Givental's expression [8] of the elliptic Gromov-Witten generating function for a smooth projective toric variety twisted by a vector bundle. The latter expression is given in terms of the equivariant Frobenius structure of the equivariant quantum cohomology. One may regard Theorem 2.6 as a mirror theorem for elliptic quasimap invariants for Calabi-Yau complete intersections in toric varieties (as well as in partial flag varieties, see Remark 2.7), in the following sense.

Whenever one computes the RHS in Conjecture 2.5 as a closed form, one obtains a closed form of the mirror theorem. Inspired by [12, 11], we accomplish the computation for Calabi-Yau complete intersections in projective spaces. This second step is completely algebraic. We will, however, see that the geometric natures of various generating functions of quasimap invariants make the step crucially simple.

1.1. Acknowledgments. We thank Ionut Ciocan-Fontanine and Jeongseok Oh for useful discussions. The research of B.K. and H.L. was partially supported by the NRF grant 2007-0093859.

\section{LocAlized Elliptic ExpRESSION}

Let $\mathbf{G}$ be a complex reductive group and let $V$ be a finite dimensional representation space of $\mathbf{G}$. Let $\theta$ be a character of $\mathbf{G}$ such that the semistable locus $V^{s s}(\theta)$ with respect to $\theta$ has no non-trivial isotropy subgroup of $\mathbf{G}$. Following the twisted theory as in [2, $\S 7$ ], we assume that a complex torus $\mathbf{T}$ acts on a vector space $V$ and this action commutes with the $\mathbf{G}$ action on $V$. Assume furthermore, the induced action on $Y:=V / /{ }_{\theta} \mathbf{G}$ allows only finitely many 0 -dimensional and 1dimensional T-orbits.

Let $E$ be a $\mathbf{G} \times \mathbf{T}$-representation space. Let $s$ be a $\mathbf{G}$-equivariant map from $V$ to $E$ whose zero locus $W$ has only locally complete intersection singularities. Assume that the semistable locus $W^{s s}(\theta)$ is nonsingular.

Denote $X=W / /{ }_{\theta} \mathbf{G}$. For $\beta \in \operatorname{Hom}_{\mathbb{Z}}\left(\operatorname{Pic}^{\mathbf{G}} V, \mathbb{Z}\right)$ let

$$
Q_{g, k}(X, \beta)\left(\operatorname{resp} . Q_{g, k}(Y, \beta)\right)
$$

be the moduli space of $k$-pointed genus $g$ stable quasimaps to $X$ (resp. $Y$ ) of class $\beta$. Denote by $f$ the universal map from the universal curve 
$\mathcal{C}$ to the stack quotient $[V / \mathbf{G}]$ :

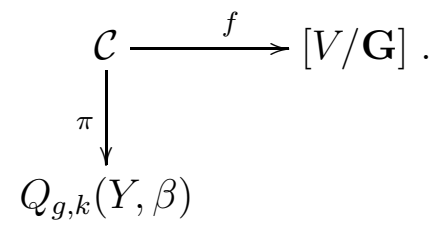

Denote

$$
\tilde{E}:=[(E \times V) / \mathbf{G}]
$$

which is a vector bundle on $[V / \mathbf{G}]$. Note that $s$ induces a section of a coherent sheaf $\pi_{*} f^{*} \tilde{E}$. Assume that for $g=0$ and also for $g=1, k=$ $0, \beta \neq 0$,

$$
R^{1} \pi_{*} f^{*} \tilde{E}=0
$$

For example this is the case when $E$ is a sum $\oplus_{a} E_{a}$ of 1-dimensional $\mathbf{G} \times \mathbf{T}$-representations $E_{a}$ with $\mathbf{G}$ weight $m_{a} \theta$ for some positive integers $m_{a}$.

Let $\iota$ denote the closed immersion of $Q_{g, k}(X, \beta)$ into $Q_{g, k}(Y, \beta)$. By the functoriality in [9] we have

$$
\iota_{*}\left[Q_{g, k}(X, \beta)\right]^{\mathrm{vir}}=\mathrm{e}\left(\pi_{*} f^{*} \tilde{E}\right) \cap\left[Q_{g, k}(Y, \beta)\right]^{\mathrm{vir}}
$$

for $g=0, k=2,3, \ldots$ and also for $g=1, k=0, \beta \neq 0$. In this paper we study $\left[Q_{1,0}(X, \beta)\right]^{\mathrm{vir}}$ using the (obvious T-equivariant version of) RHS of (2.1).

2.1. Genus zero theory. We introduce the definitions of various generating functions of rational quasimap invariants with the ordinary markings. We prove the relation (2.4) which will be needed later.

First we set the notation for the cohomology basis and its dual basis. Let $\left\{p_{i}\right\}_{i}$ be the set of $\mathbf{T}$-fixed points of $Y$ and let $\phi_{i}$ be the "delta" basis of $H_{\mathbf{T}}^{*}(Y) \otimes \mathbb{Q}(\lambda)$, that is,

$$
\left.\phi_{i}\right|_{p_{j}}= \begin{cases}1 & \text { if } i=j \\ 0 & \text { if } i \neq j\end{cases}
$$

Let $\phi^{i}$ be the dual basis with respect to the E-twisted $\mathbf{T}$-equivariant Poincaré pairing, i.e.,

$$
\int_{Y} \phi_{i} \phi^{j} \mathrm{e}^{\mathbf{T}}\left(\left.\tilde{E}\right|_{Y}\right)= \begin{cases}1 & \text { if } i=j \\ 0 & \text { if } i \neq j\end{cases}
$$

where $\mathrm{e}^{\mathbf{T}}\left(\left.\tilde{E}\right|_{Y}\right)$ is the $\mathbf{T}$-equivariant Euler class of $\left.\tilde{E}\right|_{Y}$.

We assume that, for every $i, \mathrm{e}^{\mathbf{T}}\left(\left.\tilde{E}\right|_{p_{i}}\right)$ is invertible in $\mathbb{Q}(\lambda)$ so that the twisted Poincaré pairing is a perfect pairing on $H_{\mathbf{T}}^{*}(Y) \otimes \mathbb{Q}(\lambda)$. Note 
that

$$
\phi^{i}=e_{i} \phi_{i}, \text { where } e_{i}:=\frac{1}{\int_{Y} \phi_{i} \phi_{i} \mathrm{e}^{\mathbf{T}}\left(\left.\tilde{E}\right|_{Y}\right)}=\frac{\mathrm{e}^{\mathbf{T}}\left(T_{p_{i}} Y\right)}{\mathrm{e}^{\mathbf{T}}\left(\left.\tilde{E}\right|_{p_{i}}\right)} .
$$

Integrating along the twisted virtual fundamental class

$$
\mathrm{e}^{\mathbf{T}}\left(\pi_{*} f^{*} \tilde{E}\right) \cap\left[Q_{0, k}(Y, \beta)\right]^{\mathrm{vir}}
$$

we define correlators $\langle\ldots\rangle_{0, k, \beta}^{0+}$ as follows. For $\gamma_{i} \in H_{\mathbf{T}}^{*}(Y) \otimes \mathbb{Q}(\lambda)$,

$$
\left\langle\gamma_{1} \psi^{a_{1}}, \ldots, \gamma_{k} \psi^{a_{k}}\right\rangle_{0, k, \beta}^{0+}:=\int_{\mathrm{e}^{\mathbf{T}}\left(\pi_{*} f^{*} \tilde{E}\right) \cap\left[Q_{0, k}(Y, \beta)\right]^{\mathrm{vir}}} \prod_{i} e v_{i}^{*}\left(\gamma_{i}\right) \psi_{i}^{a_{i}},
$$

where $\psi_{i}$ is the psi-class associated to the $i$-th marking and $e v_{i}$ is the $i$-th evaluation map.

Let

$$
Q_{0, k}(Y, \beta)^{\mathbf{T}, p_{i}}
$$

be the $\mathbf{T}$-fixed part of $Q_{0, k}(Y, \beta)$ whose elements have domain components only over $p_{i}$. Integrating along the localized cycle class

$$
\frac{\mathrm{e}^{\mathbf{T}}\left(\pi_{*} f^{*} \tilde{E}\right) \cap\left[Q_{0, k}(Y, \beta)^{\mathbf{T}, p_{i}}\right]^{\operatorname{vir}}}{\mathrm{e}^{\mathbf{T}}\left(N_{Q_{0, k}(Y, \beta)^{\mathbf{T}, p_{i}} / Q_{0, k}(Y, \beta)}^{\mathrm{vir}}\right)}
$$

we define $\langle\ldots\rangle_{0, k, \beta}^{0+, p_{i}}$ and $\langle\langle\ldots\rangle\rangle_{0, k}^{0+, p_{i}}$ as follows:

$$
\begin{aligned}
& \left\langle\gamma_{1} \psi^{a_{1}}, \ldots, \gamma_{k} \psi^{a_{k}}\right\rangle_{0, k, \beta}^{0+, p_{i}}:=\int_{\frac{\mathrm{e}^{\mathbf{T}}\left(\pi_{*} f^{*} \tilde{E}\right) \cap\left[Q_{0, k}(Y, \beta)\right.}{\mathrm{e}^{\mathbf{T}}\left(N_{Q_{0}}^{\mathrm{vir}} p_{i}\right]^{\mathrm{vir}}}} \prod_{i} e v_{i}^{*}\left(\gamma_{i}\right) \psi_{i}^{a_{i}} ; \\
& \left\langle\left\langle\gamma_{1} \psi^{a_{1}}, \ldots, \gamma_{k} \psi^{a_{k}}\right\rangle_{0, k}^{0+, p_{i}}\right. \\
& :=\sum_{m, \beta} \frac{q^{\beta}}{m !}\left\langle\gamma_{1} \psi^{a_{1}}, \ldots, \gamma_{k} \psi^{a_{k}}, t, \ldots, t\right\rangle_{0, k+m, \beta}^{0+, p_{i}}, \text { for } t \in H_{\mathbf{T}}^{*}(Y) \otimes \mathbb{Q}(\lambda),
\end{aligned}
$$

where $q$ is a formal Novikov variable.

In what follows, let $z$ be a formal variable. We will need the following T-local generating functions:

$$
\begin{aligned}
& D_{i}:=e_{i}\langle\langle 1,1,1\rangle\rangle_{0,3}^{0+, p_{i}}=1+O(q) ; \\
& u_{i}:=e_{i}\langle\langle 1,1\rangle\rangle_{0,2}^{0+, p_{i}}=\left.t\right|_{p_{i}}+O(q) ; \\
& S_{t}^{0+, p_{i}}(\gamma):=e_{i}\left\langle\left\langle\frac{1}{z-\psi}, \gamma\right\rangle\right\rangle_{0,2}^{0+, p_{i}}=\left.e^{t} \gamma\right|_{p_{i}}+O(q) \\
& \text { for } \gamma \in H_{\mathbf{T}}^{*}(Y) \otimes \mathbb{Q}(\lambda)[[q]] ; \\
& J^{0+, p_{i}}:=e_{i}\left\langle\left\langle\frac{1}{z(z-\psi)}\right\rangle\right\rangle_{0,1}^{0+, p_{i}}=\left.e^{t}\right|_{p_{i}}+O(q),
\end{aligned}
$$


where the unstable terms of $J^{0+, p_{i}}$ are defined by the quasimap graph spaces $Q G_{0,0, \beta}^{0+}(Y)$ as in [1, 2] so that

$$
\left.J^{0+, p_{i}}\right|_{t=0}=\left.J^{0+}\right|_{t=0, p_{i}}
$$

(see $\S 5$ of 22 for the definition of $J^{0+}$ ). Here the front terms $e_{i}$ are inserted as the class $E$-Poincaré dual to $\left.\phi_{i}\right|_{p_{i}}=1$. The parameter $z$ naturally appears as the $\mathbb{C}^{*}$-equivariant parameter in the graph construction (see $\S 4$ of $[2]$ ). It is originated from the $\mathbb{C}^{*}$-action on $\mathbb{P}^{1}$.

Denote by $Q G_{0, k, \beta}^{0+}(Y)$ the quasimap graph spaces (see [2]) and by

$$
Q G_{0, k, \beta}^{0+,}(Y)^{\mathbf{T}, p_{i}}
$$

the T-fixed part of $Q G_{0, k, \beta}(Y)$ whose elements have domain components only over $p_{i}$. Further, we define invariants and generating functions on the graph spaces: for $\gamma_{i} \in H_{\mathbf{T}}^{*}(Y) \otimes H_{\mathbb{C}^{*}}^{*}\left(\mathbb{P}^{1}\right) \otimes \mathbb{Q}(\lambda)$

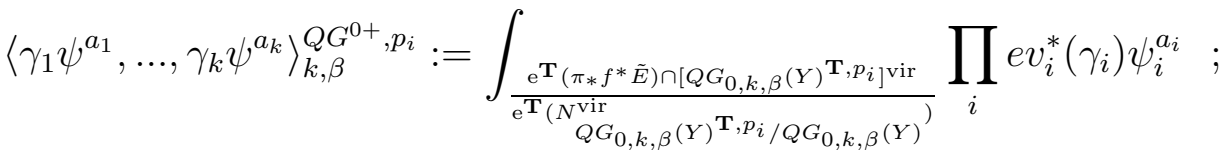

$$
\begin{aligned}
& \left\langle\left\langle\gamma_{1} \psi^{a_{1}}, \ldots, \gamma_{k} \psi^{a_{k}}\right\rangle\right\rangle_{k}^{Q G^{0+}, p_{i}} \\
& :=\sum_{m, \beta} \frac{q^{\beta}}{m !}\left\langle\gamma_{1} \psi^{a_{1}}, \ldots, \gamma_{k} \psi^{a_{k}}, t, \ldots, t\right\rangle_{k+m, \beta}^{Q G^{0+}, p_{i}} \text {, for } t \in H_{\mathbf{T}}^{*}(Y) \otimes \mathbb{Q}(\lambda) \text {. }
\end{aligned}
$$

Here we denote by $e v_{i}$ the $i$-th evaluation map to $Y \times \mathbb{P}^{1}$ from the quasimap graph spaces and regard $t$ also as the elements $t \otimes 1$ in $H_{\mathbf{T}}^{*}(Y) \otimes H_{\mathbb{C}^{*}}^{*}\left(\mathbb{P}^{1}\right) \otimes \mathbb{Q}(\lambda)$.

In what follows, let $\mathbf{p}_{\infty}$ be the equivariant cohomology class $H_{\mathbb{C}^{*}}^{*}\left(\mathbb{P}^{1}\right)$ defined by the requirements

$$
\left.\mathbf{p}_{\infty}\right|_{0}=0,\left.\mathbf{p}_{\infty}\right|_{\infty}=-z
$$

\section{Proposition 2.1.}

$$
J^{0+, p_{i}}=S_{t}^{0+, p_{i}}\left(P^{0+, p_{i}}\right)
$$

where

$$
P^{0+, p_{i}}:=e_{i}\left\langle\left\langle 1 \otimes \mathbf{p}_{\infty}\right\rangle\right\rangle_{1}^{Q G^{0+}, p_{i}}
$$

Proof. The proof is completely parallel to the poof of Theorem 5.4.1 of 2. Fix the number of markings and the degree class $\beta$ and then apply the $\mathbb{C}^{*}$-localization to the definition of $P^{0+, p_{i}}$.

By the uniqueness lemma in $\S 7.7$ of [2],

$$
S_{t}^{0+, p_{i}}(\gamma)=\left.e^{u_{i} / z} \gamma\right|_{p_{i}}
$$


Hence Proposition 2.1 gives the expression

$$
J^{0+, p_{i}}=e^{u_{i} / z}\left(r_{i, 0}+O(z)\right),
$$

where $r_{i, 0} \in \mathbb{Q}(\lambda)[[t, q]]$ is the constant term of $P^{0+, p_{i}}$ in $z$.

Corollary 2.2. The equality

$$
\log J^{0+, p_{i}}=u_{i} / z+\log r_{i, 0}+O(z) \quad \in \mathbb{Q}(\lambda)((z))[[t, q]]
$$

holds as Laurent series of z over the coefficient ring $\mathbb{Q}(\lambda)$ in each power expansion of $t$ and $q$, after regarding $t$ as a formal element.

Proof. It is clear that both side belong to $\mathbb{Q}(\lambda)((z))[[t, q]]$.

\section{Corollary 2.3.}

$$
\left.D_{i}\right|_{t=0}=\frac{1}{\left.r_{i, 0}\right|_{t=0}} .
$$

Proof. By (2.2) at $t=0,(2.3)$ with $\gamma=1$, and the definition of $J^{0+, p_{i}}$ we see that

$$
J^{0+, p_{i}}=\left.e^{\left.u_{i}\right|_{t=0} / z} P^{0+, p_{i}}\right|_{t=0}+\frac{t}{z} S_{t=0}^{0+, p_{i}}(1)+O\left(t^{2}\right) .
$$

Also by (2.3) with $\gamma=1$ and the definition of $S_{t}^{0+, p_{i}}$ we see that

$$
S_{t}^{0+, p_{i}}=e^{\left.u_{i}\right|_{t=0} / z}\left(1+\frac{t}{z}\left(\left.D_{i}\right|_{t=0}\right)\right)+O\left(t^{2}\right) .
$$

The multiplication of $e^{-\left.u_{i}\right|_{t=0} / z}$ and (2.2) after the replacements of (2.5), (2.6) gives

$$
\left.P^{0+, p_{i}}\right|_{t=0}+\frac{t}{z}+O\left(t^{2}\right)=\left.P^{0+, p_{i}}\right|_{t=0}\left(1+\left.D_{i}\right|_{t=0} \frac{t}{z}\right)+O\left(t^{2}\right) .
$$

Now the comparison of the $\frac{t}{z}$-coefficient yields (2.4).

2.2. Insertions of $0+$ weighted markings. To break the symmetry of the localization computation for the virtual fundamental classes of the elliptic quasimap moduli spaces we will need to introduce a marking. However, to keep the relation (2.1) even with markings for $g=1$, we will use the infinitesimally (i.e., $0+$ ) weighted markings.

Denote by

$$
Q_{g, k \mid m}^{0+, 0+}(Y, \beta)\left(\operatorname{resp} . Q G_{0, k \mid m, \beta}^{0+, 0+}(Y)\right)
$$

the (resp. graph) moduli space of genus $g$ (resp. genus zero), degree class $\beta$ stable quasimaps to $Y$ with ordinary $k$ pointed markings and infinitesimally weighted $m$ pointed markings (see $\S 2, \S 5$ of [4]). 
They are isomorphic to the universal curve $\mathcal{C}$ of $Q_{g, k \mid m-1}^{0+}(Y, \beta)$ (resp $\left.Q G_{0, k \mid m-1, \beta}^{0+}(Y)\right)$. Denote by

$$
Q_{g, k \mid m}^{0+, 0+}(Y, \beta)^{\mathbf{T}, p_{i}},\left(\operatorname{resp} . \quad Q G_{0, k \mid m, \beta}^{0+, 0+}(Y)^{\mathbf{T}, p_{i}}\right)
$$

the T-fixed part of $Q_{g, k \mid m}^{0+, 0+}(Y, \beta)$, (resp. $\left.Q G_{0, k \mid m, \beta}^{0+, 0+}(Y)\right)$ whose domain components are only over $p_{i}$.

For $\gamma_{i} \in H_{\mathbf{T}}^{*}(Y) \otimes \mathbb{Q}(\lambda), \tilde{t}, \delta_{j} \in H_{\mathbf{T}}^{*}([V / \mathbf{G}], \mathbb{Q})$ denote

$$
\begin{aligned}
& \left\langle\gamma_{1} \psi^{a_{1}}, \ldots, \gamma_{k} \psi^{a_{k}} ; \delta_{1}, \ldots, \delta_{m}\right\rangle_{0, k \mid m, \beta}^{0+, 0+} \\
& :=\int_{\left.\mathrm{e}^{\mathbf{T}}\left(\pi_{*} f^{*} \tilde{E}\right) \cap\left[Q_{0, k \mid m}^{0+, 0+}(Y, \beta)\right]\right]^{\mathrm{vir}}} \prod_{i} e v_{i}^{*}\left(\gamma_{i}\right) \psi_{i}^{a_{i}} \prod_{j} \hat{e v_{j}^{*}}\left(\delta_{j}\right) ; \\
& \left\langle\left\langle\gamma_{1} \psi^{a_{1}}, \ldots, \gamma_{k} \psi^{a_{k}} ; \delta_{1}, \ldots, \delta_{m}\right\rangle\right\rangle_{0, k}^{0+, 0+} \\
& :=\sum_{m^{\prime}, \beta} \frac{q^{\beta}}{m^{\prime} !}\left\langle\gamma_{1} \psi^{a_{1}}, \ldots, \gamma_{k} \psi^{a_{k}} ; \delta_{1}, \ldots, \delta_{m}, \tilde{t}, \ldots, \tilde{t}\right\rangle_{0, k \mid m+m^{\prime}, \beta}^{0+, 0+} ; \\
& \left\langle\gamma_{1} \psi^{a_{1}}, \ldots, \gamma_{k} \psi^{a_{k}} ; \delta_{1}, \ldots, \delta_{m}\right\rangle_{0, k \mid m, \beta}^{0+, 0+, p_{i}}
\end{aligned}
$$

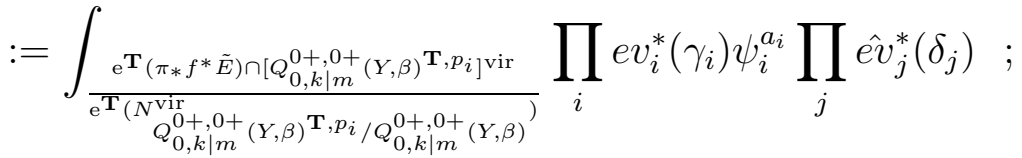

$$
\begin{aligned}
& \left\langle\left\langle\gamma_{1} \psi^{a_{1}}, \ldots, \gamma_{k} \psi^{a_{k}} ; \delta_{1}, \ldots, \delta_{m}\right\rangle\right\rangle_{0, k \mid m}^{0+, 0+, p_{i}} \\
& :=\sum_{m^{\prime}, \beta} \frac{q^{\beta}}{m^{\prime} !}\left\langle\gamma_{1} \psi^{a_{1}}, \ldots, \gamma_{k} \psi^{a_{k}} ; \delta_{1}, \ldots, \delta_{m}, \tilde{t}, \ldots, \tilde{t}\right\rangle_{0, k \mid m+m^{\prime}, \beta}^{0+, 0+, p_{i}},
\end{aligned}
$$

where $\hat{e} v_{j}$ is the evaluation map to $[V / \mathbf{G}]$ at the $j$-th infinitesimally weighted marking. Here and below by double brackets with superscript $0+, 0+$, denote the sum over all degree class $\beta$ and all possible $\tilde{t}$ insertions only at the infinitesimally weighted markings. Similarly we define

$$
\langle\cdots ; \cdots\rangle_{k \mid m, \beta}^{Q G^{0+, 0+}} \text { and }\langle\langle\cdots ; \cdots\rangle\rangle_{k \mid m}^{Q G^{0+, 0+}, p_{i}} \text {. }
$$


Consider

$$
\begin{aligned}
\mathbb{S}(\gamma) & :=\sum_{i} \phi^{i}\left\langle\left\langle\frac{\phi_{i}}{z-\psi}, \gamma\right\rangle\right\rangle_{0,2}^{0+, 0+} ; \\
\mathbb{V}_{i i}(x, y) & :=\left\langle\left\langle\frac{\phi_{i}}{x-\psi}, \frac{\phi_{i}}{y-\psi}\right\rangle\right\rangle_{0,2}^{0+, 0+}=\frac{1}{e_{i}(x+y)}+O(q) ; \\
\mathbb{U}_{i} & :=e_{i}\langle\langle 1,1\rangle\rangle_{0,2}^{0+, 0+, p_{i}}=\left.\tilde{t}\right|_{p_{i}}+O(q) ; \\
\mathbb{S}_{i}^{0+, p_{i}}(\gamma) & :=e_{i}\left\langle\left\langle\frac{1}{z-\psi}, \gamma\right\rangle\right\rangle_{0,2}^{0+, 0+, p_{i}}=\left.e^{\tilde{t} / z} \gamma\right|_{p_{i}}+O(q) ; \\
J^{0+, p_{i}} & :=e_{i}\left\langle\left\langle\frac{1}{z(z-\psi)}\right\rangle\right\rangle_{0,1}^{0+, 0+, p_{i}}=\left.e^{\tilde{t}}\right|_{p_{i}}+O(q)=\left.J^{0+, p_{i}}\right|_{t=0}+O(\tilde{t}) .
\end{aligned}
$$

(Here $e_{i}^{2} \mathbb{V}_{i i}$ at $\tilde{t}=0$ coincides with $\left.V_{t=0}^{0+}\right|_{p_{i}}$ of [3].)

As before,

$$
\begin{aligned}
\mathbb{S}_{i}^{0+, p_{i}}(\gamma) & =\left.e^{\mathbb{U}_{i} / z} \gamma\right|_{p_{i}} ; \\
\mathbb{J}^{0+, p_{i}} & =e^{\mathbb{U}_{i} / z}\left(\sum_{k=0}^{m} \mathbb{R}_{i, k} z^{k}+O\left(z^{m+1}\right)\right)
\end{aligned}
$$

for some unique $\mathbb{R}_{i, k} \in \mathbb{Q}(\lambda)[[\tilde{t}, q]]$ (after regarding $\tilde{t}$ as a formal element).

2.3. Birkhoff factorization. In this subsection we do not need to assume that the $\mathbf{T}$ action on $Y$ has isolated fixed points. Therefore in this subsection $\left\{\phi_{i}\right\}_{i}$ will denote any chosen basis of $H_{\mathbf{T}}^{*}(Y) \otimes \mathbb{Q}(\lambda)$ with its $E$-Poincaré dual basis $\left\{\phi^{i}\right\}_{i}$.

Denote by I the infinitesimal $I$-function $\mathrm{J}^{0+, 0+}$ defined in [4]. The $\mathbb{S}$ introduced in the previous section is, by the very definition, the infinitesimal $S$-operator $\mathbb{S}^{0+, 0+}$ defined in [4]. Hence

$$
\mathbb{I}:=J^{0+, 0+} \text { and } \mathbb{S}:=\mathbb{S}^{0+, 0+} \text {. }
$$

For $\gamma \in H_{\mathbf{T}}^{*}(Y), \tilde{\gamma} \in H_{\mathbf{T}}^{*}([V / \mathbf{G}])$ denotes a lift of $\gamma$, i.e., $\left.\tilde{\gamma}\right|_{Y}=\gamma$.

Let $\mathbf{p}_{0}$ be the equivariant cohomology class $H_{\mathbb{C}^{*}}^{*}\left(\mathbb{P}^{1}\right)$ defined by

$$
\left.\mathbf{p}_{0}\right|_{0}=z,\left.\mathbf{p}_{0}\right|_{\infty}=0 \text {. }
$$

Consider

$$
P_{\tilde{\gamma}}:=\sum_{i} \phi^{i}\left\langle\left\langle\phi_{i} \otimes \mathbf{p}_{\infty} ; \tilde{\gamma} \otimes \mathbf{p}_{0}\right\rangle\right\rangle_{0,1 \mid 1}^{Q G^{0+, 0+}} \in H_{\mathbf{T}}^{*}(Y) \otimes \mathbb{Q}(\lambda)[z][[\tilde{t}, q]]
$$

and its virtual $\mathbb{C}^{*}$ localization factorization. As in Proposition 4.3 of [4], there is a Birkhoff factorization

$$
z \partial_{\gamma} \mathbb{I}:=\left.z \frac{d}{d s}\right|_{s=0} \mathbb{I}(\tilde{t}+s \tilde{\gamma})=\mathbb{S}\left(P_{\tilde{\gamma}}\right) .
$$


Since $P_{\tilde{\gamma}}=\gamma+O(q)$, the factorization (2.8) implies that for each $\tilde{t}$ there is a unique expression of $\mathbb{S}(\gamma)$ as a linear combination of $\partial_{\phi_{i}}$ I with coefficients in $\mathbb{Q}(\lambda)[z][[q]]$. Hence we conclude the following Proposition.

Proposition 2.4. For each $\tilde{t}$, there are unique coefficients $a_{i}(z, q) \in$ $\mathbb{Q}(\lambda)[z][[q]]$ making

$$
\sum_{i} a_{i}(z, q) z \partial_{\phi_{i}} \mathbb{I}=\gamma+O(1 / z)
$$

Furthermore LHS coincides with $\mathbb{S}(\gamma)$.

2.4. Genus one theory. From now on, we assume that the CalabiYau condition holds, i.e.,

$$
c_{1}(Y)-c_{1}\left(\left.\tilde{E}\right|_{Y}\right)=0 \text { in } H^{2}(Y, \mathbb{Q}) .
$$

We apply Givental's localization method [8] to express a genus one generating function in terms of the genus zero generating functions.

Consider the genus one generating function with one insertion at an infinitesimally (i.e., 0+) weighted marking:

$$
\langle; \tilde{\gamma}\rangle_{1,0 \mid 1}^{0+, 0+}:=\sum_{d=1}^{\infty} q^{d}\langle; \tilde{\gamma}\rangle_{1,0 \mid 1, d}^{0+, 0+}
$$

where $\tilde{\gamma} \in H_{\mathbf{T}}^{2}([V / \mathbf{G}], \mathbb{Q})$. We will study the generating function using the virtual $\mathbf{T}$ localization.

In the following conjecture, $c_{i}(\lambda)$ denotes the element in $\mathbb{Q}(\lambda)$ uniquely determined by

$$
1+c_{i}(\lambda) \mathrm{e}(\mathbb{E})=\frac{\mathrm{e}^{\mathbf{T}}\left(\mathbb{E}^{\vee} \otimes T_{p_{i}} Y\right) \mathrm{e}^{\mathbf{T}}\left(\left.\tilde{E}\right|_{p_{i}}\right)}{\mathrm{e}^{\mathbf{T}}\left(T_{p_{i}} Y\right) \mathrm{e}^{\mathbf{T}}\left(\left.\mathbb{E}^{\vee} \otimes \tilde{E}\right|_{p_{i}}\right)},
$$

where $\mathbb{E}$ is the Hodge bundle on the moduli stack $\bar{M}_{1,1}$ of stable one pointed genus 1 curves.

Conjecture 2.5. For $\tilde{\gamma} \in H_{\mathbf{T}}^{2}([V / \mathbf{G}], \mathbb{Q})$

$$
\langle; \tilde{\gamma}\rangle_{1,0 \mid 1}^{0+, 0+}=\sum_{i} q_{\tilde{\gamma}} \frac{\partial}{\partial q_{\tilde{\gamma}}}\left(-\frac{\left.\log \mathbb{R}_{i, 0}\right|_{\tilde{t}=0}}{24}+c_{i}(\lambda) \frac{\left.\mathbb{U}_{i}\right|_{\tilde{t}=0}}{24}\right)
$$

$$
+\frac{1}{2} \sum_{i}\left(\left.\partial_{\tilde{\gamma}} \mathbb{U}_{i}\right|_{\tilde{t}=0}\right) \lim _{(x, y) \rightarrow(0,0)}\left(\left.\left(e^{-\mathbb{U}_{i}\left(\frac{1}{x}+\frac{1}{y}\right)} e_{i} \mathbb{V}_{i i}(x, y)-\frac{1}{x+y}\right)\right|_{\tilde{t}=0}\right),
$$

where $q_{\tilde{\gamma}} \frac{\partial}{\partial q_{\tilde{\gamma}}}$ acts on $q^{\beta}$ by $q_{\tilde{\gamma}} \frac{\partial}{\partial q_{\tilde{\gamma}}} q^{\beta}=q^{\beta} \int_{\beta} \tilde{\gamma}$. 
We prove Conjecture 2.5 in the following toric setting. Let $Y$ be a projective smooth toric variety defined by a fan $\Sigma$. Let $\Sigma(1)$ be the collection of all 1-dimensional cones $\rho$ in $\Sigma$ and let $V=\mathbb{C}^{\Sigma(1)}$. Then $Y$ is also given by a GIT quotient $\mathbb{C}^{\Sigma(1)} / /{ }_{\theta} \mathbf{G}$ for the complex torus $\mathbf{G}=\left(\mathbb{C}^{*}\right)^{|\Sigma(1)|-\operatorname{dim} Y}$ and some character $\theta$ of $\mathbf{G}$. Denote by $\mathbf{T}$ the big torus $\left(\mathbb{C}^{*}\right)^{\Sigma(1)}$. Let $E$ and $W$ be as in the beginning of $\$ 2$.

Theorem 2.6. Conjecture 2.5 holds true for the toric setting.

2.5. The proof of Theorem 2.6. There is a natural 1-1 correspondence between the $\mathbf{T}$ fixed points of $Y$ and the maximal cones of $\Sigma$. For a maximal cone $\sigma$, denote by $p_{\sigma}$ the corresponding $\mathbf{T}$ fixed point. The $\mathbf{T}$-fixed loci of $Q_{1,0 \mid 1}^{0+, 0+}(Y, \beta)$ are divided into two types according to whether the domain curves are irreducible or not. A quasimap in $Q_{1,0 \mid 1}^{0+, 0+}(Y, \beta)^{\mathbf{T}}$ will be called a vertex type over $p_{\sigma}$ if all domain components of the quasimap are all over $p_{\sigma}$. Otherwise, the quasimap will be called a loop type. The loop type quasimap is called a loop type over $p_{\sigma}$ if the marking of the quasimap is over $p_{\sigma}$.

Let $Q_{v e r t, \sigma}^{\mathbf{T}}$ be the substack of $Q_{1,0 \mid 1}^{0+, 0+}(Y, \beta)^{\mathbf{T}}$ consisting of the vertex types over $p_{\sigma}$. Let $Q_{\text {loop }, \sigma}^{\mathbf{T}}$ be the substack of $Q_{1,0 \mid 1}^{0+, 0+}(Y, \beta)^{\mathbf{T}}$ consisting of the loop types over $p_{\sigma}$.

By the virtual localization theorem, $\langle; \tilde{\gamma}\rangle_{1,0 \mid 1}^{0+, 0+}$ is the sum of the localization contribution Vert $\mathbf{V}_{\sigma}^{\tilde{\gamma}}$ from all the vertex types over $p_{\sigma} \in Y^{\mathbf{T}}$ and the localization contribution $\operatorname{Loop}_{\sigma}^{\tilde{\gamma}}$ from all the loop types over $p_{\sigma} \in Y^{\mathbf{T}}$. That is,

$$
\langle; \gamma\rangle_{1,0 \mid 1}^{0+, 0+}:=\sum_{\sigma} \operatorname{Vert}_{\sigma}^{\tilde{\gamma}}+\sum_{\sigma} \operatorname{Loop}_{\sigma}^{\tilde{\gamma}}
$$

where

$$
\begin{aligned}
& \operatorname{Vert}_{\sigma}^{\tilde{\gamma}}:=\sum_{\beta \neq 0} q^{\beta} \int_{\left[Q_{\text {vert }, \sigma}^{\mathbf{T}}\right]^{\mathrm{vir}}} \frac{\left.\mathrm{e}^{\mathbf{T}}\left(\pi_{*} f^{*} \tilde{E}\right)\right|_{Q_{\text {vert }, \sigma}^{\mathbf{T}}} \hat{e v_{1}^{*}(\tilde{\gamma})}}{\mathrm{e}^{\mathbf{T}}\left(N_{Q_{\text {vert }, \sigma}^{\mathrm{T}} / Q_{1,0 \mid 1}^{\text {vir }}(Y, \beta)}^{0+, 0+}\right)}, \\
& \operatorname{Loop}_{\sigma}^{\tilde{\gamma}}:=\sum_{\beta \neq 0} q^{\beta} \int_{\left[Q_{\text {loop }, \sigma}^{\mathrm{T}}\right]^{\mathrm{vir}}} \frac{\left.\mathrm{e}^{\mathbf{T}}\left(\pi_{*} f^{*} \tilde{E}\right)\right|_{Q_{\text {loop }, \sigma}^{\mathbf{T}}} \hat{e} \hat{v}_{1}^{*}(\tilde{\gamma})}{e\left(N_{Q_{\text {loop }, \sigma}^{\mathrm{T}} / Q_{1,0 \mid 1}^{0+, 0+}(Y, \beta)}^{\mathrm{vir}}\right)} .
\end{aligned}
$$

The above loop term can be identified with (2.10) by an argument completely parallel to the corresponding procedure in the proof of Theorem 2.1 of [8].

The analysis of vertex terms needs a nontrivial modification to the corresponding procedure of [8] due to the appearance of diagonal classes $\Delta_{J}$ of $\bar{M}_{1,0 \mid d}$, where $J \subset[d]:=\{1,2, \ldots, d\}$. Here $\Delta_{J}$ is the codimension 
$|J|-1$ cycle class represented by the locus where $0+$ weighted markings of $J$ coincide to each other.

Let $\operatorname{Vert}_{\sigma}$ be the $p_{\sigma}$-vertex part of \langle\rangle$_{1,0}^{0+}$. Then by the divisor axiom for the infinitesimally weighted marking, $\operatorname{Vert}_{\sigma}^{\tilde{\gamma}}=q_{\tilde{\gamma}} \frac{\partial}{\partial q_{\tilde{\gamma}}} \operatorname{Vert}_{\sigma}$. Therefore it is enough to show

$$
\operatorname{Vert}_{\sigma}=-\frac{\left.\log \mathbb{R}_{\sigma, 0}\right|_{\tilde{t}=0}}{24}+c_{\sigma}(\lambda) \frac{\left.\mathbb{U}_{\sigma}\right|_{\tilde{t}=0}}{24} .
$$

For $\rho \in \Sigma(1)$, let $\xi_{\rho}$ be the character of the $\mathbf{G}$ action on the corresponding coordinate of $\mathbb{C}^{\Sigma(1)}$. Recall that $\xi_{\rho^{\prime}}, \rho^{\prime} \not \subset \sigma$ form a basis of the character group of $\mathbf{G}$. Hence we may let $\xi_{\rho}=\sum_{\rho^{\prime} \not \subset \sigma} a_{\rho, \rho^{\prime}} \xi_{\rho^{\prime}}$ for some unique integers $a_{\rho, \rho^{\prime}}$. For a curve class $\beta \in \operatorname{Hom}_{\mathbb{Z}}\left(\mathrm{Pic}^{\mathbf{G}} V, \mathbb{Z}\right)$, denote by $\beta(\rho)$ the integer value of $\beta$ at the line bundle associated to $\xi_{\rho}$.

Let $\beta_{\sigma}$ be the set of all pairs $(\rho, j), \rho \not \subset \sigma, j \in[\beta(\rho)]$. Then the $\mathbf{T}$ fixed $p_{\sigma}$-vertex part of $Q_{1,0}(Y, \beta)$ is the quotient of $\bar{M}_{1,0 \mid \beta_{\sigma}}$ by a finite group of order $\prod_{\rho \not \subset \sigma} \beta(\rho)$ !,

For $C \in \bar{M}_{1,0 \mid \beta_{\sigma}}$, we denote the marked point by $x_{\left(\rho^{\prime}, j\right)}$ attached to the index $\left(\rho^{\prime}, j\right) \in \beta_{\sigma}$. Let $\hat{\mathbf{x}}_{\rho^{\prime}}$ for $\rho^{\prime} \not \subset \sigma$ denote the effective divisor $\sum_{j \in\left[\beta\left(\rho^{\prime}\right)\right]} x_{\left(\rho^{\prime}, j\right)}$ and let $\hat{\mathbf{x}}_{\rho}$ for $\rho \in \Sigma(1)$ denote the divisor $\sum_{\rho^{\prime}} a_{\rho, \rho^{\prime}} \hat{\mathbf{x}}_{\rho^{\prime}}$ of $C$. Here for $\rho \not \subset \sigma$ with $\beta\left(\rho^{\prime}\right)=0$ we set $\hat{\mathbf{x}}_{\rho^{\prime}}=0$. Then the corresponding quasimap in $Q_{1,0}(Y, \beta)$ is a pair

$$
\left(C,\left\{\mathcal{O}_{C}\left(\hat{\mathbf{x}}_{\rho}\right), u_{\rho}\right\}_{\rho \in \Sigma(1)}\right),
$$

where $u_{\rho}$ is the canonical section of $\mathcal{O}_{C}\left(\hat{\mathbf{x}}_{\rho}\right)$ if $\rho \not \subset \sigma$, otherwise $u_{\rho}$ is zero.

Let $r$ be the dimension of $E$. Decompose $E=\oplus_{i=1}^{r} E_{i}$ by 1-dimensional $\mathbf{T} \times \mathbf{G}$-representations $E_{i}$. Denote by $\xi_{i}$ the character of $\mathbf{G}$ associated to the $\mathbf{G}$ action on $E_{i}$. Then $\xi_{i}=\sum_{\rho^{\prime} \not \subset \sigma} b_{i, \rho^{\prime}} \xi_{\rho^{\prime}}$ for some unique integers $b_{i, \rho^{\prime}}$. Let $\hat{\mathbf{x}}_{i}=\sum_{\rho^{\prime} \not \subset \sigma} b_{i, \rho^{\prime}} \hat{\mathbf{x}}_{\rho^{\prime}}$.

In below for a divisor $D=\sum_{i} a_{i} p_{i}$ of $C$ with $p_{i} \in C, a_{i} \in \mathbb{Z}$, we define $D^{+}:=\sum_{a_{i}>0} a_{i} p_{i}$ and $D^{-}:=\sum_{a_{i}<0} a_{i} p_{i}$. By the localization formula (see $§ 5.4$ of [3]), note that

$$
\begin{gathered}
\operatorname{Vert}_{\sigma}=\sum_{d \neq 0} \frac{q^{\beta}}{\prod_{\rho \not \subset \sigma} \beta(\rho) !} \int_{\bar{M}_{1,0 \mid \beta \sigma}}\left(1+c_{\sigma}(\lambda) \mathrm{e}(\mathbb{E})\right) F_{\sigma, \beta}^{(1,0)}, \text { where } \\
F_{\sigma, \beta}^{(1,0)}=\prod_{\rho \subset \sigma} \frac{\mathrm{e}^{\mathbf{T}}\left(H^{0}\left(C, \mathcal{O}_{\hat{\mathbf{x}}_{\rho}^{-}}\left(\hat{\mathbf{x}}_{\rho}^{+}\right) \otimes \mathbb{C}_{\sigma, \rho}\right)\right.}{\mathrm{e}^{\mathbf{T}}\left(H^{0}\left(C, \mathcal{O}_{\hat{\mathbf{x}}_{\rho}^{+}}\left(\hat{\mathbf{x}}_{\rho}^{+}\right) \otimes \mathbb{C}_{\sigma, \rho}\right)\right.} \prod_{i=1}^{r} \frac{\mathrm{e}^{\mathbf{T}}\left(H^{0}\left(C,\left.\mathcal{O}_{\hat{\mathbf{x}}_{i}^{+}}\left(\hat{\mathbf{x}}_{i}^{+}\right) \otimes \tilde{E}\right|_{p_{\sigma}}\right)\right.}{\mathrm{e}^{\mathbf{T}}\left(H^{0}\left(C,\left.\mathcal{O}_{\hat{\mathbf{x}}_{i}^{-}}\left(\hat{\mathbf{x}}_{i}^{+}\right) \otimes \tilde{E}\right|_{p_{\sigma}}\right)\right.},
\end{gathered}
$$

where $\mathbb{C}_{\sigma, \rho}$ denotes the 1-dimensional $\mathbf{T}$ subspace of $T_{p_{\sigma}} Y$ corresponding to the facet of $\sigma$ complement to $\rho$. 
For nonnegative integers $g, m$, the above expression for $F_{\sigma, \beta}^{(1,0)}$ also defines $F_{\sigma, \beta}^{(g, m)}$ as an element in $H^{*}\left(\bar{M}_{g, m \mid \beta_{\sigma}}, \mathbb{Q}(\lambda)\right)$, which can be written by a polynomial of diagonal classes and the psi classes $\hat{\psi}_{k}$ (associated to the $0+$ weighted $k$-th marking).

Let $d$ be a positive integer. For $J \subset[d]$, let $\hat{\psi}_{J}$ denote $\left.\hat{\psi}_{j}\right|_{\Delta_{J}}$, for any $j \in J$. Note that for $J_{1} \cap J_{2} \neq \emptyset$,

$$
\Delta_{J_{1}} \Delta_{J_{2}}=\left(-\hat{\psi}_{J_{1} \cup J_{2}}\right)^{\left|J_{1} \cap J_{2}\right|-1} \Delta_{J_{1} \cup J_{2}}
$$

in $H^{*}\left(\bar{M}_{g, m \mid d}, \mathbb{Q}\right)$ (see $\S 4.4$ of $[10]$ ). For $j \in[d]$, define $\Delta_{j}$ to be the fundamental class. For a partition $J=\left\{J_{1}, \ldots, J_{k}\right\}$ of $[d]$ (i.e., $\emptyset \neq J_{i} \subset$ $[d]$ and $\left.\coprod_{i=1}^{k} J_{i}=[d]\right)$, define

$$
\Delta_{J}:=\Delta_{J_{1}} \ldots \Delta_{J_{k}}
$$

Then $F_{\sigma, \beta}^{(g, m)}$ can be written

$$
F_{\sigma, \beta}^{(g, m)}=\sum_{\text {partion } J=\left\{J_{1}, \ldots, J_{k}\right\} \text { of } \beta_{\sigma}} a_{J} \Delta_{J}
$$

as a linear sum of $\Delta_{J}$ over the coefficient ring $\mathbb{Q}(\lambda)\left[\hat{\psi}_{\bullet} \mid \bullet \in \beta_{\sigma}\right]$.

Since e $(\mathbb{E}) \hat{\psi}_{\bullet}=0$ in $H^{*}\left(\bar{M}_{1,0 \mid \beta_{\sigma}}\right)$ and $\hat{\psi}_{\bullet}=0$ in $H^{*}\left(\bar{M}_{0,2 \mid \beta_{\sigma}}\right)$, we see that

$$
\begin{aligned}
\int_{\bar{M}_{1,0 \mid \beta_{\sigma}}} \mathrm{e}(\mathbb{E}) F_{\sigma, \beta}^{(1,0)} & =\frac{\text { Coeff. of the const. term in } a_{\left\{\beta_{\sigma}\right\}}}{24} \\
& =\frac{1}{24} \int_{\bar{M}_{0,2 \mid \beta_{\sigma}}} F_{\sigma, \beta}^{(0,2)} .
\end{aligned}
$$

This explains the last term of (2.11).

The verification of the first term in RHS of (2.11) requires a further analysis of $F_{\sigma, \beta}^{(g, m)}$. First, observe that the $j$-th cotangent line on $\bar{M}_{g, m \mid d}$ for $j \in[d]$ is naturally isomorphic to the $j$-th cotangent line on $\bar{M}_{g, m \mid d-1}$ under the pullback of the forgetting map of the last $0+$ weighted point. Therefore $\hat{\psi}_{\bullet}^{2}=0 H^{*}\left(\bar{M}_{g, m \mid \beta_{\sigma}}\right)$ and for any partition $J=\left\{J_{1}, \ldots, J_{k}\right\}$ of $\beta_{\sigma}$

$$
\begin{aligned}
\int_{\bar{M}_{1,0 \mid \beta_{\sigma}}} \Delta_{J} \hat{\psi}_{J_{1}}^{a_{1}} \ldots \hat{\psi}_{J_{k}}^{a_{k}} & =\left\{\begin{array}{cc}
1 / 24 & \text { if } k=1, a_{1}=1 \\
0 & \text { otherwise }
\end{array} ;\right. \\
\int_{\bar{M}_{0,3 \mid \beta_{\sigma}}} \Delta_{J} \hat{\psi}_{J_{1}}^{a_{1}} \ldots \hat{\psi}_{J_{k}}^{a_{k}} & =\left\{\begin{array}{cc}
1 & \text { if } a_{1}=\ldots=a_{k}=1 \\
0 & \text { otherwise }
\end{array} .\right.
\end{aligned}
$$


Let

$$
A_{J_{1}, \ldots, J_{k}}^{\beta}:=\text { Coeff. of } \prod_{i=1}^{k} \hat{\psi}_{J_{i}} \text { in } a_{\left\{J_{1}, \ldots, J_{k}\right\}} \text {. }
$$

When $k=1$, we denote $A_{J_{1}, \ldots, J_{k}}^{\beta}$ simply by $A^{\beta}$. Denote by $\beta_{J_{i}}$ the set of all pairs $(\rho, j)$ such that $j \in\left[\left|J_{i}(\rho)\right|\right]$, where $J_{i}(\rho):=\left\{(\rho, j) \in J_{i}\right\}$. Then note that

$$
A_{J_{1}, \ldots, J_{k}}^{\beta}=\prod_{i=1}^{k} A^{\beta_{J_{i}}}
$$

which follows from two properties:

1) $F_{\sigma, \beta}$ is a product of the $\mathbf{T}$-equivariant Euler classes of vector bundles with fibers $H^{0}\left(C, \mathcal{O}_{D}(B)\right)$ where $D$ is an effective divisor and $B$ is a divisor of $C$. Here supports of $D$ and $B$ are contained in $\beta_{\sigma}$.

2) Let $D=D_{1}+D_{2}$, where $D_{1}, D_{2}$ are effective and let $B=B_{1}+B_{2}$. Then in the K-group element

$\mathcal{O}_{D_{1}+D_{2}}\left(B_{1}+B_{2}\right)=\mathcal{O}_{D_{1}}\left(B_{1}\right) \otimes \mathcal{O}_{D_{1}}\left(B_{2}\right)+\mathcal{O}_{D_{2}}\left(B_{2}\right) \otimes \mathcal{O}_{D_{2}}\left(-D_{1}+B_{1}\right)$

Suppose that $\beta_{\sigma}$ is a disjoint union of $S_{1}, S_{2}$ such that supports of $D_{i}$, $B_{i}$ are in $S_{i}$ for each $i=1,2$. Then

$$
\begin{aligned}
\left.\mathrm{e}^{\mathbf{T}}\left(H^{0}\left(C, \mathcal{O}_{D_{1}+D_{2}}\left(B_{1}+B_{2}\right)\right)\right)\right|_{S_{1}, S_{2}} \\
\quad=\mathrm{e}^{\mathbf{T}}\left(H^{0}\left(C, \mathcal{O}_{D_{1}}\left(B_{1}\right)\right)\right) \mathrm{e}^{\mathbf{T}}\left(H^{0}\left(C, \mathcal{O}_{D_{2}}\left(B_{2}\right)\right)\right),
\end{aligned}
$$

where the restriction to $S_{1}, S_{2}$ is defined to be letting $\Delta_{J}=0$ whenever there is $J_{i}$ in the partition $J$ such that $J_{i}$ intersects with $S_{1}$ and $S_{2}$ simultaneously. 
By (2.12), (2.13), (2.14) we note that

$$
\begin{aligned}
& \int_{\bar{M}_{0,3 \mid \beta \sigma}} F_{\sigma, \beta}^{(0,3)}=\sum_{k=1}^{\infty} \sum_{\substack{\text { partition } J_{1}, \ldots, J_{k} \\
\text { of } \beta_{\sigma}}} \prod_{i=1}^{k} A^{\beta_{J_{i}}} \\
& =\sum_{k=1}^{\infty} \frac{1}{k !} \sum_{\substack{\text { ordered partition } \\
\left(J_{1}, \ldots, J_{k}\right) \text { of } \beta_{\sigma}}} \prod_{i=1}^{k} A^{\beta_{J_{i}}} \\
& =\sum_{k=1}^{\infty} \frac{1}{k !} \sum_{\substack{\left(\beta_{J_{1}}, \ldots, \beta_{J_{k}}\right) \\
\text { ordered }}} \prod_{\rho \not \subset \sigma}\left(J_{1}(\rho)|, \ldots,| J_{k}(\rho) \mid\right) \prod_{i=1}^{k} A^{\beta_{J_{i}}} \\
& =\prod_{\rho \not \subset \sigma} \beta(\rho) ! \sum_{k=1}^{\infty} \frac{1}{k !} \sum_{\substack{\text { ordered } \\
\left(\beta_{J_{1}}, \ldots, \beta_{J_{k}}\right)}} \prod_{i=1}^{k}\left(\frac{24}{\prod_{\rho \not \subset \sigma}\left|J_{i}(\rho)\right| !} \int_{\bar{M}_{1,0 \mid \beta}} F_{\sigma_{J_{i}} \beta_{J_{i}}}^{(1,0)}\right) .
\end{aligned}
$$

Hence

$$
\sum_{\beta \neq 0} \frac{q^{\beta}}{\prod_{\rho \not \subset \sigma} \beta(\rho) !} \int_{\bar{M}_{1,0 \mid \beta \sigma}} F_{\sigma, \beta}^{(1,0)}=\frac{\left.\log D_{\sigma}\right|_{t=0}}{24} .
$$

This combined with (2.4) verifies the first term in RHS of (2.11).

Remark 2.7. By $§ 5.9 .2$ of [3], it is clear that the above proof works also for Calabi-Yau zero loci of homogeneous vector bundles on partial flag varieties $Y$, local toric varieties, local Grassmannians, and the total spaces of the cotangent bundles of partial flag varieties.

\section{Explicit Computations}

In this section we prove Theorem 1.1. From now on unless stated otherwise, let $\mathbf{G}=\mathbb{C}^{*}, \mathbf{T}=\left(\mathbb{C}^{*}\right)^{n}$, and let $\mathbb{C}_{l_{a}}$ be the 1-dimensional representation space of $\mathbf{G}$ with positive weight $l_{a}$. Let $E=\bigoplus_{a=1}^{r} \mathbb{C}_{l_{a}}$, with $\sum_{a=1}^{r} l_{a}=n$. We take the standard $\mathbf{T}$-action on $V$ and the $\mathbf{T}$ trivial action on $E$. This gives rise to a $\mathbf{T}$-equivariant vector bundle $\tilde{E}$ on $[V / \mathbf{G}]$. Choose a character $\theta$ such that $Y:=V / /{ }_{\theta} \mathbf{G}$ becomes $\mathbb{P}^{n-1}$. Under the natural isomorphism $\operatorname{Hom}_{\mathbb{Z}}\left(\mathrm{Pic}^{\mathrm{G}} V, \mathbb{Z}\right) \cong \mathbb{Z}$, we use a nonnegative integer $d$ instead of $\beta$. Let $p_{i}$ be the $i$-th $\mathbf{T}$ fixed point of $Y$ as in (1.2).

3.1. Birkhoff factorization revisited. By [7] (see also (5.3.1) of [4]),

$$
\mathbb{I}_{\tilde{t}=0}=\left.I_{\mathbf{T}}\right|_{t=0}
$$

where LHS and RHS are defined in $\$ 2.3$ and (1.1), respectively. 
We define the degrees of $\lambda, H, q$ as

$$
\operatorname{deg} \lambda_{j}=1=\operatorname{deg} H, \operatorname{deg} q=0 .
$$

Then it is easy to check that, for $k=0,1, \ldots, n-1$, the $1 / z^{k}$-coefficient $I_{k}$ of

$$
\left.I_{\mathbf{T}}\right|_{t=0}
$$

is a homogeneous degree $k$ element in $\mathbb{Q}\left[\sigma_{1}, \ldots, \sigma_{n-1}, H\right][[q]]$ satisfying

$$
I_{k} \in \mathbb{Q}[[q]] H^{k} \text { modulo (1.3). }
$$

On the other hand

$$
\mathbb{S}_{\tilde{t}=0}\left(H^{k}\right)=H^{k}+O(1 / z) \text { for } 0 \leq k \leq n-1 .
$$

Throughout $\$ 3$ we impose the condition (1.3). After [12] we define an operation as follows.

For $F \in\left(\frac{\mathbb{Q}[H]}{\left(H^{n}-\lambda_{0}^{n}\right)}\right)[[1 / z]][[q]]$ with $\left.\left(\left(z q \frac{d}{d q}+H\right) F\right)\right|_{H=1, z=\infty, q=0} \neq 0$

let

$$
\mathfrak{B}(F):=\frac{\left(z q \frac{d}{d q}+H\right) F(z, H, q)}{\left.\left(\left(z q \frac{d}{d q}+H\right) F(z, H, q)\right)\right|_{H=1, z=\infty}} .
$$

Consider $\mathfrak{B}^{k}\left(\frac{\left.\underline{I}_{\mathbf{T}}\right|_{t=0}}{I_{0}}\right)$ and note that it is of form $H^{k}+O(1 / z)$ and homogenous of degree $k$ if we put $\operatorname{deg} z=1$.

\section{Corollary 3.1.}

$$
\mathbb{S}_{\tilde{t}=0}\left(H^{k}\right) \equiv \mathfrak{B}^{k}\left(\frac{\left.\underline{I}_{\mathbf{T}}\right|_{t=0}}{I_{0}}\right), k=0,1, \ldots, n-1 .
$$

Here we recall that $\equiv$ denotes the equality modulo relations (1.3).

Proof. Let $\tilde{H} \in H_{\mathbf{T}}^{*}\left(\left[\mathbb{C}^{n} / \mathbb{C}^{*}\right]\right)$ be the natural lift of $H$ and let

$$
\tilde{t}=\sum_{i=0}^{n-1} t_{i} \tilde{H}^{i}
$$

with formal variables $t_{i}$. Then, there is the ( $\mathbf{T}$-equivariant version of) derivative form formula of the big I-function as in $\S 5.3$ of [4]:

$$
\mathbb{I}(\tilde{t})=\left.\left(\exp \left(\sum_{i=0}^{n-1} \frac{t_{i}}{z}\left(z q \frac{d}{d q}+H\right)^{i}\right)\right) I_{\mathbf{T}}\right|_{t=0},
$$

which shows that

$$
\left.\left(z \partial_{H^{i}} \mathbb{I}\right)\right|_{\tilde{t}=0}=\left(z q \frac{d}{d q}+H\right)^{i}\left(\left.\mathbb{I}\right|_{\tilde{t}=0}\right) .
$$


By (3.3) and Proposition 2.4, in order to verify (3.2), it is enough to recall that both sides of (3.2) are of form $H^{k}+O(1 / z)$.

Now consider an equivariant cohomology basis

$$
\left\{1, H:=c_{1}^{\mathbf{T}}(\mathcal{O}(1)), \ldots, H^{n-1}\right\}
$$

of the T-equivariant cohomology ring

$$
H_{\mathbf{T}}^{*}\left(\mathbb{P}^{n-1}\right) \cong \mathbb{Q}\left[\lambda_{1}, \ldots, \lambda_{n}, h\right] /\left(\prod_{i=1}^{n}\left(h-\lambda_{i}\right)\right), \quad H \mapsto h .
$$

Its E-twisted Poincaré metric modulo relations (1.3) becomes,

$$
g_{i j}:=\left\{\begin{aligned}
\prod_{a} l_{a} & \text { if } i+j=n-1-r \\
\lambda_{0}^{n} \prod_{a} l_{a} & \text { if } i+j=2 n-1-r
\end{aligned}\right.
$$

for $0 \leq i, j \leq n-1$. Here we use the relation $H^{n}=-\prod_{j=1}^{n}\left(-\lambda_{j}\right)=\lambda_{0}^{n}$.

There is an expression of $V$-correlators in terms of $S$-correlators by Theorem 3.2 .1 of [3]:

$$
\left.e_{i} \mathbb{V}_{i i}(x, y)\right|_{\tilde{t}=0}=\frac{1}{e_{i}} \frac{\left.\left.\sum_{j} \mathbb{S}_{z=x, \tilde{t}=0}\left(\phi_{j}\right)\right|_{p_{i}} \mathbb{S}_{z=y, \tilde{t}=0}\left(\phi^{j}\right)\right|_{p_{i}}}{x+y}
$$

Hence

$$
\begin{gathered}
\left.e_{i} \mathbb{V}_{i i}\right|_{\tilde{t}=0} \equiv \frac{1}{\left(\prod l_{a}\right) e_{i}(x+y)}\left(\left.\left.\sum_{k=0}^{n-1-r} \mathbb{S}_{z=x, \tilde{t}=0}\left(H^{k}\right)\right|_{p_{i}} \mathbb{S}_{z=y, \tilde{t}=0}\left(H^{n-1-r-k}\right)\right|_{p_{i}}\right. \\
\left.+\left.\left.\frac{1}{\lambda_{0}^{n}} \sum_{b=0}^{r-1} \mathbb{S}_{z=x, \tilde{t}=0}\left(H^{n-r+b}\right)\right|_{p_{i}} \mathbb{S}_{z=y, \tilde{t}=0}\left(H^{n-1-b}\right)\right|_{p_{i}}\right) .
\end{gathered}
$$

3.2. Vertex terms. Applying (2.7) to

$$
\left.\mathbb{I}\right|_{\tilde{t}=0, p_{i}}=\left.\left.I_{\mathbf{T}}\right|_{t=0, p_{i}} \equiv \sum_{d=0}^{\infty} q^{d} \frac{\prod_{a=1}^{r} \prod_{k=1}^{l_{a} d}\left(l_{a}+k \frac{z}{\lambda_{i}}\right)}{\prod_{k=1}^{d}\left(\left(1+k \frac{z}{\lambda_{i}}\right)^{n}-1\right)} \equiv I_{\mathbf{T}}\right|_{t=0, p_{n}, z \mapsto z / \lambda_{i}},
$$

we obtain

$$
\left.\mathbb{I}\right|_{\tilde{t}=0, p_{i}} \equiv e^{\mu(q) \lambda_{i} / z}\left(\sum_{k=0}^{\infty} R_{k}(q)\left(z / \lambda_{i}\right)^{k}\right)
$$

for some $\mu(q) \in q \mathbb{Q}[[q]]$ and $R_{k}(q) \in \mathbb{Q}[[q]]$. Hence

$$
\begin{aligned}
\left.\mathbb{I}\right|_{\tilde{t}=t_{H} \tilde{H}, p_{i}} & \equiv e^{\lambda_{i} t_{H} / z}\left(\left.I_{\mathbf{T}}\right|_{t=0, q \mapsto q e^{t} H}\right) \\
& \equiv e^{\lambda_{i} t_{H} / z} e^{\mu\left(q e^{t_{H}}\right) \lambda_{i} / z}\left(\sum_{k=0}^{\infty} R_{k}\left(q e^{t_{H}}\right)\left(z / \lambda_{i}\right)^{k}\right) .
\end{aligned}
$$


Thus

$$
\begin{aligned}
\left.\mathbb{U}_{i}\right|_{\tilde{t}=t_{H} \tilde{H}} & \equiv \lambda_{i}\left(t_{H}+\mu\left(q e^{t_{H}}\right)\right) \text { and } \\
\mathbb{R}_{i, k} & \equiv R_{k}\left(q e^{t_{H}}\right) /\left(\lambda_{i}\right)^{k} .
\end{aligned}
$$

Since

$$
\begin{aligned}
\left.r_{i, 0}\right|_{t=0} & =\left.\mathbb{R}_{i, 0}\right|_{\tilde{t}=0}, \\
\left.u_{i}\right|_{t=0} & =\left.\mathbb{U}_{i}\right|_{\tilde{t}=0}, \text { and } \\
c_{i}(\lambda) & =\left(\sum_{j \neq i} \frac{1}{\lambda_{j}-\lambda_{i}}\right)+\sum_{a} \frac{1}{l_{a} \lambda_{i}},
\end{aligned}
$$

we conclude that

$$
\begin{aligned}
\sum_{i} \frac{\left.\log D_{i}\right|_{t=0}}{24} & \equiv \frac{-n \log R_{0}(q)}{24} \\
\sum_{i} \frac{\left.c_{i}(\lambda) u_{i}\right|_{t=0}}{24} & \equiv \frac{1}{24}\left(\sum_{a} \frac{n}{l_{a}}-\left(\begin{array}{l}
n \\
2
\end{array}\right) \mu(q) .\right.
\end{aligned}
$$

3.3. Loop terms. If we let

$$
\begin{aligned}
\mathrm{W}_{p, p^{\prime}} & :=\left.\left.\left((\mathfrak{B})^{p} \frac{\left.\underline{I}_{\mathbf{T}}\right|_{t=0}}{I_{0}}\right)\right|_{H=1, z=x}\left((\mathfrak{B})^{p^{\prime}} \frac{\left.\underline{I}_{\mathbf{T}}\right|_{t=0}}{I_{0}}\right)\right|_{H=1, z=y} ; \\
\mathrm{V}(x, y, q) & :=\sum_{p+p^{\prime}=n-1-r} \mathrm{~W}_{p, p^{\prime}}+\sum_{p+p^{\prime}=2 n-1-r, n-r \leq p \leq n-1} \mathrm{~W}_{p, p^{\prime}},
\end{aligned}
$$

then by (3.2) and (3.4),

$$
\left.e_{i} \mathbb{V}_{i i}(x, y)\right|_{\tilde{t}=0} \equiv \frac{\lambda_{i}^{n-1-r}}{\left(\prod l_{a}\right) e_{i}(x+y)} \mathrm{V}\left(x / \lambda_{i}, y / \lambda_{i}, q\right) .
$$

Here we use also the degree property that $\operatorname{deg}\left(\mathbb{S}\left(\phi_{j}\right) \mathbb{S}\left(\phi^{j}\right)\right)=n-1-r$.

Therefore

$$
\begin{aligned}
e^{-\left.\mathbb{U}_{i}\right|_{\tilde{t}=0}(1 / x+1 / y)} e_{i} & \left.\mathbb{V}_{i i}(x, y)\right|_{\tilde{t}=0} \\
& \equiv \frac{\lambda_{i}^{n-1}}{\mathrm{e}^{\mathbf{T}}\left(T_{p_{i}} Y\right)(x+y)} e^{-\mu(q)\left(\lambda_{i} / x+\lambda_{i} / y\right)} \mathrm{V}\left(x / \lambda_{i}, y / \lambda_{i}, q\right) .
\end{aligned}
$$

Now the limit of $x, y \rightarrow 0$ of (2.10) (or equivalently the residue at $x=0, y=0$ of $\left.\frac{(2.10)}{x y}\right)$ as computed in [11, Lemma 5.4] becomes $\left.\lim _{(x, y) \rightarrow(0,0)}\left(e^{-\mathbb{U}_{i}(1 / x+1 / y)} e_{i} \mathbb{V}_{i i}(x, y)-\frac{1}{x+y}\right)\right|_{\tilde{t}=0} \equiv \frac{\lambda_{i}^{n-2}}{\mathrm{e}^{\mathbf{T}}\left(T_{p_{i}} Y\right) L(q)} q \frac{d}{d q} \operatorname{Loop}(q)$, 
where

$$
\begin{aligned}
& L(q):=\left(1-q \prod_{a} l_{a}^{l_{a}}\right)^{-1 / n} \text { and } \\
& \operatorname{Loop}(q):=\frac{n}{24}\left(n-1-2 \sum_{a=1}^{r} \frac{1}{l_{a}}\right) \mu(q) \\
& -\frac{3(n-1-r)^{2}+(n-2)}{24} \log \left(1-q \prod l_{a}^{l_{a}}\right)-\sum_{k=0}^{n-2-r}\left(\begin{array}{c}
n-r-k \\
2
\end{array}\right) \log C_{k}(q) .
\end{aligned}
$$

Since

$$
\left.\frac{\partial \mathbb{U}_{i}}{\partial t_{\gamma}}\right|_{\tilde{t}=0}=L(q) \lambda_{i}
$$

by (3.5) and Proposition 3.2 below, we conclude that

$$
\sum_{i} \mathbf{L o o p}_{i}=\frac{1}{2} q \frac{d}{d q} \operatorname{Loop}(q) .
$$

3.4. Proof of Theorem 1.1. Now the sum

$$
(3.6)+(3.7)+\frac{1}{2} \operatorname{Loop}(q)
$$

can be explicitly obtained by Proposition 3.2 and hence we complete the proof of Theorem 1.1.

3.5. Explicit computations of $\mu, R_{0}, R_{1}$ and loop terms. Recall we assume (1.3). Let $\lambda_{0}=1$. Note that $I_{\mathbf{T}}$ satisfies the differential equation (see [7, Corollary 11.7])

$$
\left.\mathrm{PF} I_{\mathbf{T}}\right|_{t=t_{H} \cdot H}=0, \quad \mathrm{PF}:=\left(z \frac{d}{d t}\right)^{n}-1-q \prod_{a} \prod_{m=1}^{l_{a}}\left(l_{a} z \frac{d}{d t}+m z\right) .
$$

Applying the differential operator PF to the asymptotic form of

$$
\left.I_{\mathbf{T}}\right|_{t=t_{H} H, p_{n}}
$$

one obtain $\mu, R_{0}, R_{1}$ and the loop limit (see $\S 4.2, \S 4.3$ of [11] for details).

For the reader's convenience, we state the following Proposition due to Popa [11.

Proposition 3.2. [11, Proposition $4.3 \& 4.4]$

Consider $C_{b}, b=0,1, \ldots, n-1$.

(1) $\prod_{i=0}^{n-r} C_{i}=\left(1-q \prod_{a} l_{a}^{l_{a}}\right)^{-1}$.

(2) $C_{b}=C_{n-r-b}$ for $b=0,1, \ldots, n-r$.

(3) $C_{b}=1$ for $b=n-r+1, \ldots, n-1$. 
(4)

$$
\begin{gathered}
\mu(q)=\int_{0}^{q} \frac{\left.\left.\left(1-x \prod_{a} l_{a}^{l_{a}}\right)\right)\right)^{-\frac{1}{n}}-1}{x} d x, \\
R_{0}=L^{\frac{r+1}{2}} .
\end{gathered}
$$

Remark 3.3. Let $\left\{\left(H^{b}\right)^{\vee}\right\}_{b}$ be the E-twisted Poincaré dual basis of $\left\{H^{b}\right\}_{b}$. Note that

$$
C_{b} \equiv\left\langle\left(H^{b}\right)^{\vee}, H^{b-1} ; H\right\rangle_{0,2 \mid 1}^{0+, 0+}, \text { for } b=1, \ldots, n-1
$$

and hence item (2) of Proposition 3.2 naturally follows except the claim $C_{0}=C_{n-r}$.

\section{REFERENCES}

[1] I. Ciocan-Fontanine and B. Kim, Moduli stacks of stable toric quasimaps, Adv. in Math. 225 (2010), 3022-3051.

[2] I. Ciocan-Fontanine and B. Kim, Wall-crossing in genus zero quasimap theory and mirror maps, Algebr. Geom. 1 (2014), no. 4, 400-448.

[3] I. Ciocan-Fontanine and B. Kim, Higher genus quasimap wall-crossing for semi-positive targets, arXiv:1308.6377, To appear in Journal of the European Mathematical Society.

[4] I. Ciocan-Fontanine and B. Kim, Big I-functions, arXiv:1401.7417, To appear in Proceedings of the conference on the occasion of Mukai's 60th birthday.

[5] I. Ciocan-Fontanine and B. Kim, In preparation.

[6] I. Ciocan-Fontanine, B. Kim, and D. Maulik, Stable quasimaps to GIT quotients, J. Geom. Phys. 75 (2014), 17-47.

[7] A. Givental, Equivariant Gromov-Witten invariants, Internat. Math. Res. Notices 1996, no. 13, 613-663.

[8] A. Givental, Elliptic Gromov-Witten invariants and the generalized mirror conjecture, Integrable systems and algebraic geometry (Kobe/Kyoto, 1997), 107-155, World Sci. Publ., River Edge, NJ, 1998.

[9] B. Kim, A. Kresch, and T. Pantev, Functoriality in intersection theory and a conjecture of Cox, Katz, and Lee, J. Pure Appl. Algebra 179 (2003), no. 1-2, $127-136$.

[10] A. Marian, D. Oprea, and R. Pandharipande, The moduli space of stable quotients, Geom. Topol. 15 (2011), no. 3, 1651-1706.

[11] A. Popa The genus one Gromov-Witten invariants of Calabi-Yau complete intersections, Trans. Amer. Math. Soc. 365 (2013), no. 3, 1149-1181.

[12] A. Zinger, The reduced genus 1 Gromov-Witten invariants of Calabi-Yau hypersurfaces, J. Amer. Math. Soc. 22 (2009), 691-737. 
School of Mathematics, Korea Institute for Advanced Study, 85 Hoegiro, Dongdaemun-gu, Seoul, 02455, Korea

E-mail address: bumsig@kias.re.kr

School of Mathematics, Korea Institute for Advanced Study, 85 Hoegiro, Dongdaemun-gu, Seoul, 02455, Korea

E-mail address: hyenho@kias.re.kr 